\title{
Uniqueness of $q$-shift difference polynomials of meromorphic functions sharing a small function
}

\author{
Renukadevi S. Dyavanal and Ashwini M. Hattikal \\ Department of Mathematics, Karnatak University, Dharwad, India
}

Received: 23 August 2016, Accepted: 17 December 2016

Published online: 26 March 2017.

\begin{abstract}
In this article, we study the uniqueness problems of $q$-shift difference polynomials of meromorphic functions $f$ and $g$ sharing a small function $a(z)$. The results in this paper extend the results of F.H.Liu and H.X.Yi.
\end{abstract}

Keywords: Meromorphic functions, q-shift Difference polynomials, small function.

\section{Introduction and main results}

Let $\mathbb{C}$ denote the complex plane and $f$ be a non-constant meromorphic function in $\mathbb{C}$. We shall use the standard notations in the Nevanlinna's value distribution theory of meromorphic functions such as $T(r, f), N(r, f), \bar{N}(r, f)$ and $m(r, f)$, as explained in Yang and Yi[14], L.Yang [13] and Hayman[7]. The notation $S(r, f)$ is defined to be any quantity satisfying $S(r, f)=o(T(r, f))$, as $r \rightarrow \infty$ possibly outside a set $r$ of finite linear measure. A meromorphic function $a(z)$ is called a small function with respect to $f(z)$, provided that $T(r, a(z))=S(r, f)$. The following definitions are useful in proving our main results.

Definition 1. We denote and define order of $f(z)$ by

$$
\rho(f)=\limsup _{r \rightarrow \infty} \frac{\log T(r, f)}{\log r}
$$

If a non-constant meromorphic function $f(z)$ is of zero order, then $\rho(f)=0$.

Definition 2. Let ' $a$ ' be a finite complex number and $k$ be a positive integer. We denote by $N_{k)}(r, 1 /(f-a))$ the counting function for the zeros of $f(z)-a$ in $|z| \leq r$ with multiplicity $\leq k$ and by $\bar{N}_{k)}(r, 1 /(f-a))$ the corresponding one for which multiplicity is not counted. Let $N_{(k}(r, 1 /(f-a))$ be the counting function for the zeros of $f(z)-a$ in $|z| \leq r$ with multiplicity $\geq k$ and by $\bar{N}_{(k}(r, 1 /(f-a))$ the corresponding one for which multiplicity is not counted. Then we have

$$
N_{k}(r, 1 /(f-a))=\bar{N}_{(1}(r, 1 /(f-a))+\bar{N}_{(2}(r, 1 /(f-a))+\ldots+\bar{N}_{(k}(r, 1 /(f-a))
$$

Definition 3. Let $f(z)$ and $g(z)$ be two meromorphic functions in the complex plane $\mathbb{C}$. Let a $(z)$ is a small function with respect to $f$ and $g$. If $f(z)-a(z)$ and $g(z)-a(z)$ assume the same zeros with the same multiplicities, then we say that $f(z)$ and $g(z)$ share the value' $a(z)^{\prime} C M$.

For a meromorphic function $f(z)$ and a non-zero constant $c, f(z+c)$ is called the shift of $f$, for a non-zero complex constant $q, f(q z+c)$ is called the $q$-shift of $f(z)$, such that $f(z)$ is not periodic function with period $c$. And also product of difference as $\prod_{j=1}^{d} f\left(q_{j} z+c_{j}\right)^{s_{j}}$ where $d, \lambda\left(=\sum_{j=1}^{d} s_{j}\right.$ for $\left.j=1,2 \ldots, d\right)$ are positive integers and $q_{j}, c_{j}(j=1,2, \ldots d)$ are distinct non-zero complex constants.

A number of papers have focused on value distribution and uniqueness of difference polynomials, which are analogues 
of value distribution and uniqueness of differential polynomials of meromorphic functions in Nevanlinna theory.

In 2014, X.M.Li, H.X.Yi and W.L.Li[8] proved the following theorem on uniqueness of difference polynomials of meromorphic functions sharing a small function.

Theorem 1. Let $f$ and $g$ be two transcedental meromorphic functions of finite order, let $\alpha \neq \equiv$ be an entire function such that $\rho(\alpha)<\rho(f)$, let $\eta$ be a non-zero complex number and let $n$ and $m$ be two positive integers such that $n \geq m+12$ and $m \geq 2$. Suppose $f^{n}(z)\left(f^{m}(z)-1\right) f(z+\eta)-\alpha(z)$ and $g^{n}(z)\left(g^{m}(z)-1\right) g(z+\eta)-\alpha(z)$ share $0, \infty C M$. Then $f(z)=\operatorname{tg}(z)$, where $t$ is a constant satisfying $t^{m}=1$.

Further, K.Y.Zhang and H.X.Yi[16] extended the result of X.M.Li, H.X.Yi and W.L.Li[8] and proved the theorem on uniqueness of product of differential-difference polynomials of entire functions as in the following theorem.

Theorem 2. Let $f(z)$ and $g(z)$ be transcendental entire functions of finite order, $\alpha(z) \not \equiv 0$ be a common small function with respect to $f$ and $g, c_{j}(j=1,2, \ldots, d)$ be distinct finite complex numbers and $n, m, d$ and $v_{j}(j=1,2, \ldots, d)$ are nonnegative integers. If $n \geq 4 k-m+\sigma+9$ and the differential-difference polynomial $\left(f^{n}(z)(f(z)-1)^{m} \prod_{j=1}^{d} f\left(z+c_{j}\right)^{v_{j}}\right)^{(k)}$ and $\left(g^{n}(z)(g(z)-1)^{m} \prod_{j=1}^{d} g\left(z+c_{j}\right)^{v_{j}}\right)^{(k)}$ share $\alpha(z) C M$, then $f \equiv g$.

Recently, F.H.Liu and H.X.Yi[10] improved the previous results by considering uniqueness problems on product of difference polynomials of meromorphic functions.

Theorem 3. Let $f(z)$ and $g(z)$ be non-constant meromorphic functions satisfying $\rho(f)<\infty, \rho(g)<\infty$. $f(z)$ and $g(z)$ share $\infty I M . \alpha(z) \not \equiv 0$ is an entire function satisfying $\rho(\alpha)<\rho(f) . m, n, s, \mu_{j}(j=1,2 \ldots, s)$ are non-negative integers, $\sigma=\sum_{j=1}^{s} \mu_{j} . c_{j}(j=1,2 \ldots, s)$ are non-zero complex constants. $F(z)=f^{n}\left(f^{m}-1\right) \prod_{j=1}^{s} f\left(z+c_{j}\right)^{\mu_{j}}, G(z)=g^{n}\left(g^{m}-\right.$ 1) $\prod_{j=1}^{s} g\left(z+c_{j}\right)^{\mu_{j}}$ share $\alpha, \infty C M$. If $n \geq m+2 s+3 \sigma+7$ we get $f(z)=\operatorname{tg}(z)$, where $t$ is a constant satisfying $t^{m}=1$.

In this article, we investigate on uniqueness of difference polynomials of meromorphic functions sharing a small function $a(z)$ with counting multiplicity.

Theorem 4. Let $f$ and $g$ be two non-constant meromorphic functions of zero order and a $z$ ) is a small function with respect to both $f$ and $g$. Let $n \geq m+3 \lambda+2 d+7$ be a positive integer, where $m, d, \lambda\left(=\sum_{j=1}^{d} s_{j}\right.$ for $\left.j=1,2 \ldots, d\right)$ are finite positive integers such that $d<\lambda$. Let $q_{j}, c_{j}(j=1,2, \ldots d)$ are distinct non-zero complex constants. If

$$
f^{n}(z)(f(z)-1)^{m} \prod_{j=1}^{d} f\left(q_{j} z+c_{j}\right)^{s_{j}}
$$

and

$$
g^{n}(z)(g(z)-1)^{m} \prod_{j=1}^{d} g\left(q_{j} z+c_{j}\right)^{s_{j}}
$$

share $a(z) C M, f$ and $g$ share $\infty I M$, then

(1) if $m \geq 2$, then either $f=\operatorname{tg}$ for a constant $t$ such that $t^{d}=1$ where $d=G C D(n+m+\lambda, n+m+\lambda-1, \ldots, n+m+$ $\lambda-i, \ldots, n+\lambda)$ or $f$ and $g$ satisfy the algebraic equation $R(f, g) \equiv 0$, where

$$
R\left(\omega_{1}, \omega_{2}\right)=\omega_{1}^{n}\left(\omega_{1}-1\right)^{m} \prod_{j=1}^{d} \omega_{1}\left(q_{j} z+c_{j}\right)^{s_{j}}-\omega_{2}^{n}\left(\omega_{2}-1\right)^{m} \prod_{j=1}^{d} \omega_{2}\left(q_{j} z+c_{j}\right)^{s_{j}}
$$

(2) if $m=1$, then $f=$ tg for a constant such that $t^{d}=1$ where $d=G C D(n+\lambda, n+1+\lambda)$.

Also, the result in the Theorem 1. holds for entire functions for $n \geq \lambda+m+4$.

Corollary 1. Let $f$ and $g$ be two non-constant entire functions of zero order. Let $n \geq \lambda+m+4$ be a positive integer where $d, \lambda\left(=\sum_{j=1}^{d} s_{j}\right.$ for $\left.j=1,2 \ldots, d\right)$ are positive integers. Let $q_{j}, c_{j}(j=1,2, \ldots d)$ are distinct non-zero complex constants. If $f^{n}(z)(f(z)-1)^{m} \prod_{j=1}^{d} f\left(q_{j} z+c_{j}\right)^{s_{j}}$ and $g^{n}(z)(g(z)-1)^{m} \prod_{j=1}^{d} g\left(q_{j} z+c_{j}\right)^{s_{j}}$ share $a(z) C M$, $f$ and $g$ share $\infty$ IM, then

(1) if $m \geq 2$, then either $f=\operatorname{tg}$ for a constant $t$ such that $t^{d}=1$ where $d=G C D(n+m+\lambda, n+m+\lambda-1, \ldots, n+m+$ $\lambda-i, \ldots, n+\lambda)$ or $f$ and $g$ satisfy the algebraic equation $R\left(\omega_{1}, \omega_{2}\right) \equiv 0$, where

$$
R\left(\omega_{1}, \omega_{2}\right)=\omega_{1}^{n}\left(\omega_{1}-1\right)^{m} \prod_{j=1}^{d} \omega_{1}\left(q_{j} z+c_{j}\right)^{s_{j}}-\omega_{2}^{n}\left(\omega_{2}-1\right)^{m} \prod_{j=1}^{d} \omega_{2}\left(q_{j} z+c_{j}\right)^{s_{j}}
$$


(2) if $m=1$, then $f=\operatorname{tg}$ for a constant $t$ such that $t^{d}=1$ where $d=G C D\{n+\lambda, n+1+\lambda\}$.

\section{Some lemmas}

Lemma 1. [14] Let $f(z)$ be a non-constant meromorphic function, and $a_{n}(\neq 0), a_{n-1}, \ldots, a_{0}$ be small functions with respect to $f$. Then

$$
T\left(r, a_{n} f^{n}+a_{n-1} f^{n-1}+\ldots+a_{1} f+a_{0}\right)=n T(r, f)+S(r, f)
$$

Lemma 2. [12] Let $f_{1}, f_{2}$ and $f_{3}$ be non-constant meromorphic functions such that $f_{1}+f_{2}+f_{3}=1$. If $f_{1}, f_{2}$ and $f_{3}$ are linearly independent, then

$$
T\left(r, f_{1}\right)<\sum_{i=1}^{3} N_{2}\left(r, \frac{1}{f_{i}}\right)+\sum_{i=1}^{3} N_{2}\left(r, f_{i}\right)+o(T(r)),
$$

where $T(r)=\max _{1 \leq i \leq 3}\left\{T\left(r, f_{i}\right)\right\}$ and $r \notin E$.

Lemma 3. [15] Let $f_{1}$ and $f_{2}$ be two non-constant meromorphic functions. If $c_{1} f_{1}+c_{2} f_{2}=c_{3}$, where $c_{1}, c_{2}$ and $c_{3}$ are non-zero constants, then

$$
T\left(r, f_{1}\right) \leq \bar{N}\left(r, f_{1}\right)+\bar{N}\left(r, \frac{1}{f_{1}}\right)+\bar{N}\left(r, \frac{1}{f_{2}}\right)+S\left(r, f_{1}\right)
$$

Lemma 4. [11] Let $f(z)$ be a non-constant meromorphic function of zero order, and let c and $q$ be two non-zero complex numbers. Then

$$
T(r, f(q z+c)) \leq T(r, f(z))+S(r, f),
$$

on a set of logarithmic density 1.

Lemma 5. [9] Let $f$ be a meromorphic function with zero order and $c$ and $q$ be two non-zero complex numbers. Then

$$
\begin{array}{ll}
N\left(r, \frac{1}{f(q z+c)}\right) \leq N\left(r, \frac{1}{f(z)}\right)+S(r, f) & N(r, f(q z+c)) \leq N(r, f)+S(r, f) \\
\bar{N}\left(r, \frac{1}{f(q z+c)}\right) \leq N\left(r, \frac{1}{f(z)}\right)+S(r, f) & \bar{N}(r, f(q z+c)) \leq N(r, f)+S(r, f)
\end{array}
$$

outside of a possible exceptional set $E$ with finite logarithmic measure.

Lemma 6. Let $f$ be a transcendental meromorphic function of zero order and $F(z)=f^{n}(f-1)^{m} \prod_{j=1}^{d} f\left(q_{j} z+c_{j}\right)^{s_{j}}$. Then

$$
(n+m-\lambda) T(r, f)+S(r, f) \leq T(r, F) \leq(n+m+\lambda) T(r, f)+S(r, f)
$$

Proof. From Lemma 1 and Lemma 4, we have

$$
\begin{aligned}
T\left(r, f^{n}(f-1)^{m} \prod_{j=1}^{d} f\left(q_{j} z+c_{j}\right)^{s_{j}}\right) & \leq T\left(r, f^{n}\right)+T\left(r,(f-1)^{m}\right)+T\left(r, \prod_{j=1}^{d} f\left(q_{j} z+c_{j}\right)^{s_{j}}\right)+S(r, f) \\
& \leq(n+m+\lambda) T(r, f)+S(r, f) .
\end{aligned}
$$

On the other hand, from Lemma 1 and Lemma 5, we have

$$
\begin{aligned}
(n+m+\lambda) T(r, f) & =T\left(r, f^{n}(f-1)^{m} f^{\lambda}\right)+S(r, f) \\
& \leq m\left(r, \frac{F f^{\lambda}}{\prod_{j=1}^{d} f\left(q_{j} z+c_{j}\right)^{s_{j}}}\right)+N\left(r, \frac{F f^{\lambda}}{\prod_{j=1}^{d} f\left(q_{j} z+c_{j}\right)^{s_{j}}}\right)+S(r, f) \\
& \leq m(r, F)+N(r, F)+m\left(r, \frac{f^{\lambda}}{\prod_{j=1}^{d} f\left(q_{j} z+c_{j}\right)^{s_{j}}}\right)+N\left(r, \frac{f^{\lambda}}{\prod_{j=1}^{d} f\left(q_{j} z+c_{j}\right)^{s_{j}}}\right)+S(r, f) \\
& \leq T(r, F)+2 \lambda T(r, f)+S(r, f) \\
(n+m-\lambda) T(r, f)+S(r, f) & \leq T(r, F) .
\end{aligned}
$$


Lemma 7. Let $f(z)$ and $g(z)$ be non-constant meromorphic functions of zero order. Let $f^{n}(f-1)^{m} \prod_{j=1}^{d} f\left(q_{j} z+c_{j}\right)^{s_{j}}$ and $g^{n}(g-1)^{m} \prod_{j=1}^{d} g\left(q_{j} z+c_{j}\right)^{s_{j}}$ share $a(z) C M$ and $n>m+3 \lambda+2 d+7$ is a positive integer. Then

$$
T(r, g) \leq\left(\frac{n+m+\lambda}{n+m-2 d-\lambda-3}\right) T(r, f)+\log r+S(r, g) .
$$

Proof. Let $F=f^{n}(f-1)^{m} \prod_{j=1}^{d} f\left(q_{j} z+c_{j}\right)^{s_{j}}$ and $G=g^{n}(g-1)^{m} \prod_{j=1}^{d} g\left(q_{j} z+c_{j}\right)^{s_{j}}$. Applying Nevanlinna's second fundamental theorem to $G$, we have

$$
\begin{aligned}
T(r, G) & \leq \bar{N}(r, G)+\bar{N}\left(r, \frac{1}{G}\right)+\bar{N}\left(r, \frac{1}{G-a(z)}\right)+S(r, G) \\
& \leq(d+1) T(r, g)+(d+2) T(r, g)+\bar{N}\left(r, \frac{1}{G-a(z)}\right)+S(r, g)
\end{aligned}
$$

By the Lemma 1, (4) and hypothesis, we have

$$
\begin{aligned}
(n+m) T(r, g) & \leq T\left(r, g^{n}(g-1)^{m}\right)+S(r, g) \\
& \leq T\left(r, g^{n}(g-1)^{m} \prod_{j=1}^{d} g\left(q_{j} z+c_{j}\right)^{s_{j}}\right)+T\left(r, \frac{1}{\prod_{j=1}^{d} g\left(q_{j} z+c_{j}\right)^{s_{j}}}\right)+S(r, g) \\
& =T(r, G)+T\left(r, \frac{1}{\prod_{j=1}^{d} g\left(q_{j} z+c_{j}\right)^{s_{j}}}\right)+S(r, g) \\
& \leq(d+1) T(r, g)+(d+2) T(r, g)+N\left(r, \frac{1}{G-a(z)}\right)+\lambda T(r, g)+S(r, g) \\
& \leq(d+1) T(r, g)+(\lambda+d+2) T(r, g)+\bar{N}\left(r, \frac{1}{F-a(z)}\right)+S(r, g)
\end{aligned}
$$

By first fundamental theorem, we have

$$
\begin{aligned}
\bar{N}\left(r, \frac{1}{F-a(z)}\right) & \leq T\left(r, \frac{1}{F-a(z)}\right)=T(r, F-a(z))+S(r, g) \\
& =T\left(r, f^{n}(f-1)^{m} \prod_{j=1}^{d} f\left(q_{j} z+c_{j}\right)^{s_{j}}-a(z)\right)+S(r, g) \\
& \leq n T(r, f)+m T(r, f-1)+\lambda T(r, f)+\log r+S(r, g)
\end{aligned}
$$

From (5) and (6), we have

$$
\begin{aligned}
(n+m) T(r, g) & \leq(d+1) T(r, g)+(\lambda+d+2) T(r, g)+n T(r, f)+m T(r, f-1)+\lambda T(r, f)+S(r, g) \\
& \leq(2 d+3+\lambda) T(r, g)+(n+m+\lambda) T(r, f)+S(r, g) \\
(n+m-2 d-\lambda-3) T(r, g) & \leq(n+m+\lambda) T(r, f)+S(r, g) \\
T(r, g) & \leq\left(\frac{n+m+\lambda}{n+m-2 d-\lambda-3}\right) T(r, f)+S(r, g)
\end{aligned}
$$

Hence we get Lemma 7.

The following lemma is required to prove our main result.

Lemma 8. Let $f(z)$ and $g(z)$ be two non-constant meromorphic functions with zero order. Let $n \geq 3 \lambda+2 d+8$ be a positive integer, where $d, \lambda\left(=\sum_{j=1}^{d} s_{j}\right.$ for $\left.j=1,2 \ldots, d\right)$ are positive integers. Let $q_{j}, c_{j}(j=1,2, \ldots d)$ are distinct nonzero complex constants. If $f^{n}(z)(f(z)-1) \prod_{j=1}^{d} f\left(q_{j} z+c_{j}\right)^{s_{j}}=g^{n}(z)(g(z)-1) \prod_{j=1}^{d} g\left(q_{j} z+c_{j}\right)^{s_{j}}$ then we get $f=$ t $g$ for a constant $t$ such that $t^{d}=1$ where $d=G C D(n+\lambda, n+1+\lambda)$. 
Proof.

$$
f^{n}(z)(f(z)-1) \prod_{j=1}^{d} f\left(q_{j} z+c_{j}\right)^{s_{j}}=g^{n}(z)(g(z)-1) \prod_{j=1}^{d} g\left(q_{j} z+c_{j}\right)^{s_{j}}
$$

Let $h=\frac{f}{g}$, then substituting $f=g h$ in $(8)$, we get

$$
\begin{aligned}
& g^{n}(z) h^{n}(g h-1) \prod_{j=1}^{d} g\left(q_{j} z+c_{j}\right)^{s_{j}} \prod_{j=1}^{d} h\left(q_{j} z+c_{j}\right)^{s_{j}}=g^{n}(z)(g(z)-1) \prod_{j=1}^{d} g\left(q_{j} z+c_{j}\right)^{s_{j}} . \\
\Rightarrow & \left(g^{n+1} h^{n+1}-g^{n} h^{n}\right) \prod_{j=1}^{d} g\left(q_{j} z+c_{j}\right)^{s_{j}} \prod_{j=1}^{d} h\left(q_{j} z+c_{j}\right)^{s_{j}}-\left(g^{n+1}-g^{n}\right) \prod_{j=1}^{d} g\left(q_{j} z+c_{j}\right)^{s_{j}}=0 . \\
\Rightarrow & {\left[g^{n+1}\left(h^{n+1} \prod_{j=1}^{d} h\left(q_{j} z+c_{j}\right)^{s_{j}}-1\right)-g^{n}\left(h^{n} \prod_{j=1}^{d} h\left(q_{j} z+c_{j}\right)^{s_{j}}-1\right)\right] \prod_{j=1}^{d} g\left(q_{j} z+c_{j}\right)^{s_{j}}=0 . }
\end{aligned}
$$

If $h$ is not a constant, then $h$ is a non-constant meromorphic function. From (9), we have

$$
g=\frac{h^{n} \prod_{j=1}^{d} h\left(q_{j} z+c_{j}\right)^{s_{j}}-1}{h^{n+1} \prod_{j=1}^{d} h\left(q_{j} z+c_{j}\right)^{s_{j}}-1}
$$

Next, we show that 1 is not the Picard exceptional value of $H_{1}=h^{n+1} \prod_{j=1}^{d} h\left(q_{j} z+c_{j}\right)^{s_{j}}$, for that we suppose 1 is the Picard exceptional value of $H_{1}$. By the second fundamental theorem, we have

$$
\begin{aligned}
T\left(r, h^{n+1} \prod_{j=1}^{d} h\left(q_{j} z+c_{j}\right)^{s_{j}}\right) & \leq \bar{N}\left(r, h^{n+1} \prod_{j=1}^{d} h\left(q_{j} z+c_{j}\right)^{s_{j}}\right) \\
& +\bar{N}\left(r, \frac{1}{h^{n+1} \prod_{j=1}^{d} h\left(q_{j} z+c_{j}\right)^{s_{j}}}\right)+\bar{N}\left(r, \frac{1}{h^{n+1} \prod_{j=1}^{d} h\left(q_{j} z+c_{j}\right)^{s_{j}}-1}\right)+S(r, h) \\
& \leq \bar{N}(r, h)+\bar{N}\left(r, \prod_{j=1}^{d} h\left(q_{j} z+c_{j}\right)^{s_{j}}\right)+\bar{N}\left(r, \frac{1}{h}\right)+\bar{N}\left(r, \frac{1}{\prod_{j=1}^{d} h\left(q_{j} z+c_{j}\right)^{s_{j}}}\right)+S(r, h) \\
T\left(r, h^{n+1} \prod_{j=1}^{d} h\left(q_{j} z+c_{j}\right)^{s_{j}}\right) & \leq(2+2 d) T(r, h)+S(r, h) .
\end{aligned}
$$

On the other hand from first fundamental theorem and (11), we have

$$
\begin{aligned}
(n+1) T(r, h) & =T\left(r, h^{n+1}\right)+S(r, h) \\
& \leq T\left(r, h^{n+1} \prod_{j=1}^{d} h\left(q_{j} z+c_{j}\right)^{s_{j}}\right)+T\left(r, \frac{1}{\prod_{j=1}^{d} h\left(q_{j} z+c_{j}\right)^{s_{j}}}\right)+S(r, h) \\
& \leq T\left(r, h^{n+1} \prod_{j=1}^{d} h\left(q_{j} z+c_{j}\right)^{s_{j}}\right)+T\left(r, \prod_{j=1}^{d} h\left(q_{j} z+c_{j}\right)^{s_{j}}\right)+S(r, h) \\
(n+1) T(r, h) & \leq(2+2 d+\lambda) T(r, h)+S(r, h) .
\end{aligned}
$$

This is contradiction to the fact that $n \geq 3 \lambda+2 d+8>\lambda+2 d+1$. Therefore 1 is not the Picard exceptional value of $H_{1}$. Hence there exist $z_{0}$ such that $H_{1}\left(z_{0}\right)=h\left(z_{0}\right)^{n+1} \prod_{j=1}^{d} h\left(q_{j} z_{0}+c_{j}\right)^{s_{j}}=1$. For further proof we consider the following two cases.

Case 1. If $H_{1} \not \equiv 1$, then either $h(z)$ is a rational function or $h(z)$ is a transcendental meromorphic function. If $h(z)$ is a rational function. From (10), we obtain that $g(z)$ is also a rational function. This is contradiction to the condition $g(z)$ is a non-constant transcendental meromorphic function. Hence $h(z)$ is a transcendental meromorphic function. Since $f(z)$ 
and $g(z)$ are trancendental meromorphic function of zero order, $h(z)$ is also trancendental meromorphic function of zero order. Let $H_{2}=h^{n} \prod_{j=1}^{d} h\left(q_{j} z+c_{j}\right)^{s_{j}}$ and $z_{0} \in \mathbb{C}$ is the 1-point of $H_{1}$, but not the 1-point of $H_{2}$. From (10), we can get that $z_{0}$ is the zero of $H_{1}-1$ with multiplicity atleast 1 . Now assume that $z_{1} \in \mathbb{C}$ is the common zero of $H_{1}-1$ and $H_{2}-1$. Then from (10), we have

$$
\left(h^{n+1}\left(z_{1}\right) \prod_{j=1}^{d} h\left(q_{j} z_{1}+c_{j}\right)^{s_{j}}-1\right) g=h^{n}\left(z_{1}\right) \prod_{j=1}^{d} h\left(q_{j} z_{1}+c_{j}\right)^{s_{j}}-1
$$

$h^{n+1}\left(z_{1}\right) \prod_{j=1}^{d} h\left(q_{j} z_{1}+c_{j}\right)^{s_{j}}=1$ and $h^{n}\left(z_{1}\right) \prod_{j=1}^{d} h\left(q_{j} z_{1}+c_{j}\right)^{s_{j}}=1$. Then we have from (13) that $h\left(z_{1}\right)=1$. Hence

$$
\begin{aligned}
\bar{N}\left(r, \frac{1}{H_{1}-1}\right) & \leq \bar{N}\left(r, \frac{1}{H_{1}-1=0, H_{2}-1 \neq 0}\right)+\bar{N}\left(r, \frac{1}{h-1}\right)+S(r, h) \\
& \leq \bar{N}\left(r, \frac{1}{h^{n+1} \prod_{j=1}^{d} h\left(q_{j} z+c_{j}\right)^{s_{j}}-1}\right)+\bar{N}\left(r, \frac{1}{h-1}\right)+S(r, h) \\
& \leq(d+1) T(r, h)+T(r, h)+S(r, h) \\
& \leq(d+2) T(r, h)+S(r, h),
\end{aligned}
$$

where $\bar{N}\left(r, \frac{1}{H_{1}-1=0, H_{2}-1 \neq 0}\right)$ denote the counting function of zeros of $H_{1}(z)-1$ in $|z|<r$, such that each point in this is not a zero of $H_{2}(z)-1$ and each such point is counted with ignore its multiplicity. Since $h$ is a meromorphic function with zero order implies $H_{1}$ is also a meromorphic function with zero order. Thus, we have

$$
\begin{aligned}
(n+1+\lambda) T(r, h) & =T\left(r, h^{n+1+\lambda}\right)+S(r, h) \\
& \leq T\left(r, H_{1}\right)+T\left(r, \frac{h^{n+1+\lambda}}{H_{1}}\right)+S(r, h) \\
& \leq T\left(r, H_{1}\right)+T\left(r, \frac{h^{\lambda}}{\prod_{j=1}^{d} h\left(q_{j} z+c_{j}\right)^{s_{j}}}\right)+S(r, h) .
\end{aligned}
$$

By second fundamental theorem and (14), we have

$$
\begin{aligned}
T\left(r, H_{1}\right) & \leq \bar{N}\left(r, H_{1}\right)+\bar{N}\left(r, \frac{1}{H_{1}}\right)+\bar{N}\left(r, \frac{1}{H_{1}-1}\right)+S\left(r, H_{1}\right) \\
& \leq \bar{N}\left(r, h^{n+1} \prod_{j=1}^{d} h\left(q_{j} z+c_{j}\right)^{s_{j}}\right)+\bar{N}\left(r, \frac{1}{h^{n+1} \prod_{j=1}^{d} h\left(q_{j} z+c_{j}\right)^{s_{j}}}\right)+S(r, h)+\bar{N}\left(r, \frac{1}{h^{n+1} \prod_{j=1}^{d} h\left(q_{j} z+c_{j}\right)^{s_{j}}-1}\right)+S(r, h) \\
& \leq(d+1) \bar{N}(r, h)+(d+1) \bar{N}(r, h)+(d+2) T(r, h)+S(r, h) \\
& \leq(3 d+4) T(r, h)+S(r, h) .
\end{aligned}
$$

From (15) and (16), we have

$$
\begin{aligned}
(n+1+\lambda) T(r, h) & \leq(3 d+4) T(r, h)+2 \lambda T(r, h)+S(r, h) \\
& \leq(2 \lambda+3 d+4) T(r, h)+S(r, h) .
\end{aligned}
$$

Which is contradiction to $n \geq 3 \lambda+2 d+8>\lambda+3 d+3$.

Case 2. If $H_{1} \equiv 1$, then

$$
\begin{aligned}
(n+1) T(r, h) & =T\left(r, h^{n+1}\right)=T\left(r, h^{n+1} \prod_{j=1}^{d} h\left(q_{j} z+c_{j}\right)^{s_{j}}\right)+T\left(r, \frac{1}{\prod_{j=1}^{d} h\left(q_{j} z+c_{j}\right)^{s_{j}}}\right)+S(r, h) \\
& \leq T(r, 1)+T\left(r, \prod_{j=1}^{d} h\left(q_{j} z+c_{j}\right)^{s_{j}}\right)+S(r, h) \\
& \leq \lambda T(r, h)+S(r, h) .
\end{aligned}
$$


Which is contradiction to $n \geq 3 \lambda+2 d+8>\lambda-1$. Hence $h$ is a constant, then from (10), we have $h^{d}=1$ where $d=G C D(n+1+\lambda, n+\lambda)$. Hence proved the Lemma (8).

Proof. (Proof of Theorem 1.) Let $F(z)=f^{n}(z)(f(z)-1)^{m} \prod_{j=1}^{d} f\left(q_{j} z+c_{j}\right)^{s_{j}}$ and $G(z)=g^{n}(z)(g(z)-1)^{m} \prod_{j=1}^{d} g\left(q_{j} z+\right.$ $\left.c_{j}\right)^{s_{j}}$. By the hypothesis we have $F(z)$ and $G(z)$ share $a(z)$ CM and $f, g$ share $\infty$ IM. Let

$$
H=\frac{f^{n}(z)(f(z)-1)^{m} \prod_{j=1}^{d} f\left(q_{j} z+c_{j}\right)^{s_{j}}-a(z)}{g^{n}(z)(g(z)-1)^{m} \prod_{j=1}^{d} g\left(q_{j} z+c_{j}\right)^{s_{j}}-a(z)}
$$

then, $H$ is a meromorphic function and $H \not \equiv 0$. Therefore, we have

$$
\begin{aligned}
T(r, H) & =T\left(r, \frac{f^{n}(z)(f(z)-1)^{m} \prod_{j=1}^{d} f\left(q_{j} z+c_{j}\right)^{s_{j}}-a(z)}{g^{n}(z)(g(z)-1)^{m} \prod_{j=1}^{d} g\left(q_{j} z+c_{j}\right)^{s_{j}}-a(z)}\right) \\
& \leq T\left(r, f^{n}(z)(f(z)-1)^{m} \prod_{j=1}^{d} f\left(q_{j} z+c_{j}\right)^{s_{j}}-a(z)\right)+T\left(r, g^{n}(z)(g(z)-1)^{m} \prod_{j=1}^{d} g\left(q_{j} z+c_{j}\right)^{s_{j}}-a(z)\right)+O(1) \\
& \leq T\left(r, f^{n}\right)+T\left(r,(f-1)^{m}\right)+T\left(r, \prod_{j=1}^{d} f\left(q_{j} z+c_{j}\right)^{s_{j}}\right)+T\left(r, g^{n}\right)+T\left(r,(g-1)^{m}\right) \\
& +T\left(r, \prod_{j=1}^{d} f\left(q_{j} z+c_{j}\right)^{s_{j}}\right)+O(\log r) \\
& \leq(n+m+\lambda)[T(r, f)+T(r, g)]+O(\log r) .
\end{aligned}
$$

Therefore $T(r, H) \leq O[T(r, f)+T(r, g)]$.

From (17), it is clear that the zeros and poles of $H$ are multiple and also the following inequalities satisfies obviously.

$$
\begin{gathered}
\bar{N}(r, H) \leq \bar{N}_{L}(r, f) \text { and } \bar{N}\left(r, \frac{1}{H}\right) \leq \bar{N}_{L}(r, g) \\
\bar{N}_{L}(r, f)+\bar{N}_{L}(r, g) \leq \bar{N}(r, f)=\bar{N}(r, g)
\end{gathered}
$$

Let

$$
F_{1}=\frac{f^{n}(f-1)^{m} \prod_{j=1}^{d} f\left(q_{j} z+c_{j}\right)^{s_{j}}}{a(z)} ; F_{2}=H ; F_{3}=\frac{-H g^{n}(g-1)^{m} \prod_{j=1}^{d} g\left(q_{j} z+c_{j}\right)^{s_{j}}}{a(z)}
$$

such that $F_{1}+F_{2}+F_{3}=1$. Let $T(r)$ denote the maximum of $T\left(r, F_{j}\right)$ for $j=1,2,3 . \therefore$ we have $T\left(r, F_{1}\right)=O(T(r, f))$, $T\left(r, F_{2}\right)=O(T(r, f)+T(r, g))$ and $T\left(r, F_{3}\right)=O(T(r, f)+T(r, g))$. Thus, we also have $S(r, f)+S(r, g)=o(T(r))$. Now we discuss the three possible cases separately.

Case 3. If possible, suppose that neither $F_{2}$ nor $F_{3}$ is a constant.

If $F_{1}, F_{2}$, and $F_{3}$ are linearly independent, then by Lemma (2) and (20), we have

$$
\begin{aligned}
T\left(r, F_{1}\right) & <\sum_{i=1}^{3} N_{2}\left(r, \frac{1}{F_{i}}\right)+\sum_{i=1}^{3} \bar{N}\left(r, F_{i}\right)+o(T(r)) \\
& \leq N_{2}\left(r, \frac{a(z)}{f^{n}(f-1)^{m} \prod_{j=1}^{d} f\left(q_{j} z+c_{j}\right)^{s_{j}}}\right)+N_{2}\left(r, \frac{1}{H}\right)+N_{2}\left(r, \frac{a(z)}{-H g^{n}(g-1)^{m} \prod_{j=1}^{d} g\left(q_{j} z+c_{j}\right)^{s_{j}}}\right) \\
& +\bar{N}\left(r, \frac{f^{n}(f-1)^{m} \prod_{j=1}^{d} f\left(q_{j} z+c_{j}\right)^{s_{j}}}{a(z)}\right)+\bar{N}(r, H)+\bar{N}\left(r, \frac{-H g^{n}(g-1)^{m} \prod_{j=1}^{d} g\left(q_{j} z+c_{j}\right)^{s_{j}}}{a(z)}\right)+o(T(r)) .
\end{aligned}
$$


Since $H \neq 0, \forall z \in \mathbb{C}$, we have $N_{2}\left(r, \frac{1}{H}\right)=0$. Then (21) becomes

$$
\begin{aligned}
T\left(r, F_{1}\right) & \leq N\left(r, \frac{1}{f^{n}(f-1)^{m} \prod_{j=1}^{d} f\left(q_{j} z+c_{j}\right)^{s_{j}}}\right)-N_{(3}\left(r, \frac{1}{f^{n}(f-1)^{m} \prod_{j=1}^{d} f\left(q_{j} z+c_{j}\right)^{s_{j}}}\right) \\
& +2 \bar{N}_{(3}\left(r, \frac{1}{f^{n}(f-1)^{m} \prod_{j=1}^{d} f\left(q_{j} z+c_{j}\right)^{s_{j}}}\right)+N\left(r, \frac{1}{g^{n}(g-1)^{m} \prod_{j=1}^{d} g\left(q_{j} z+c_{j}\right)^{s_{j}}}\right) \\
& -N_{(3}\left(r, \frac{1}{g^{n}(g-1)^{m} \prod_{j=1}^{d} g\left(q_{j} z+c_{j}\right)^{s_{j}}}\right)+2 \bar{N}_{(3}\left(r, \frac{1}{g^{n}(g-1)^{m} \prod_{j=1}^{d} g\left(q_{j} z+c_{j}\right)^{s_{j}}}\right) \\
& +\bar{N}(r, f)+\bar{N}\left(r, \prod_{j=1}^{d} f\left(q_{j} z+c_{j}\right)^{s_{j}}\right)+\bar{N}(r, H)+\bar{N}(r,-H)+\bar{N}(r, g) \\
& +\bar{N}\left(r, \prod_{j=1}^{d} g\left(q_{j} z+c_{j}\right)^{s_{j}}\right)+2 \log r+o(T(r)) \\
& \leq(n+m+\lambda) N\left(r, \frac{1}{f}\right)-(n-2) N\left(r, \frac{1}{f}\right)+(n+m+\lambda) N\left(r, \frac{1}{g}\right)-(n-2) N\left(r, \frac{1}{g}\right) \\
& +(d+1) \bar{N}(r, f)+2 \bar{N}_{L}(r, f)+(d+1) \bar{N}(r, g)+2 \log r+o(T(r))
\end{aligned}
$$

From Lemma (6), we have

$$
\begin{aligned}
(n+m-\lambda) T(r, f) & <T\left(r, F_{1}\right) \leq(n+m+\lambda-n+2+d+1) T(r, f)+2 \bar{N}_{L}(r, f) \\
& +(n+m+\lambda-n+2+d+1) T(r, g)+o(T(r)) \\
& <(\lambda+m+d+3) T(r, f)+2 \bar{N}_{L}(r, f)+(\lambda+m+d+3) T(r, g)+o(T(r))
\end{aligned}
$$

Let

$$
G_{1}=\frac{g^{n}(g-1)^{m} \prod_{j=1}^{d} g\left(q_{j} z+c_{j}\right)^{s_{j}}}{a(z)} ; \quad G_{2}=\frac{1}{H} ; \quad G_{3}=-\frac{f^{n}(f-1)^{m} \prod_{j=1}^{d} f\left(q_{j} z+c_{j}\right)^{s_{j}}}{H a(z)}
$$

such that $G_{1}+G_{2}+G_{3}=1$. In the same manner as for $F_{1}, F_{2}, F_{3}$, we get

$$
(n+m-\lambda) T(r, g)<(\lambda+m+d+3) T(r, g)++2 \bar{N}_{L}(r, g)+(\lambda+m+d+3) T(r, f)+o(T(r))
$$

From (19),(22) and (23), we have

$$
\begin{aligned}
(n+m-\lambda)[T(r, f)+T(r, g)] & <(\lambda+m+d+3)[T(r, f)+T(r, g)]+2 \bar{N}_{L}(r, f)+2 \bar{N}_{L}(r, g) \\
& +(\lambda+m+d+3)[T(r, f)+T(r, g)]+S(r, f)+S(r, g) \\
& <(\lambda+m+d+3)[T(r, f)+T(r, g)]+2 \bar{N}(r, f)+(\lambda+m+d+3)[T(r, f)+T(r, g)] \\
& +S(r, f)+S(r, g) \\
& <(\lambda+m+d+3)[T(r, f)+T(r, g)]+T(r, f)+T(r, g)+(\lambda+m+d+3)[T(r, f)+T(r, g)] \\
& +S(r, f)+S(r, g) \\
& <(2 \lambda+2 m+2 d+7)[T(r, f)+T(r, g)]+S(r, f)+S(r, g) .
\end{aligned}
$$

Which is contradiction to $n>3 \lambda+m+2 d+7$. Thus our assumption $F_{1}, F_{2}$ and $F_{3}$ are linearly independent is wrong. Hence $F_{1}, F_{2}, F_{3}$ are linearly dependent. Thus, there exists three constants $\left(c_{1}, c_{2}, c_{3}\right) \neq(0,0,0)$, such that

$$
c_{1} F_{1}+c_{2} F_{2}+c_{3} F_{3}=0
$$

If $c_{1}=0\left[c_{2} \neq 0 \& c_{3} \neq 0\right]$, from (24), we have

$$
\begin{gathered}
F_{3}=\frac{-c_{2}}{c_{3}} F_{2} \\
\Rightarrow g^{n}(g-1)^{m} \prod_{j=1}^{d} g\left(q_{j} z+c_{j}\right)^{s_{j}}=\frac{c_{2}}{c_{3}} a(z) .
\end{gathered}
$$


From Lemma 6, we have

$$
(n+m-\lambda) T(r, g) \leq \log r+O(1) .
$$

Since $n>3 \lambda+m+2 d+7$, we get a contradiction. Thus $c_{1} \neq 0$ and by (24), we have

$$
F_{1}=\frac{-c_{2}}{c_{1}} F_{2}-\frac{c_{3}}{c_{1}} F_{3}
$$

Since $F_{1}+F_{2}+F_{3}=1$, we get

$$
\left(1-\frac{c_{2}}{c_{1}}\right) F_{2}+\left(1-\frac{c_{3}}{c_{1}}\right) F_{3}=1
$$

Where $c_{1} \neq c_{2}, c_{1} \neq c_{3}$. From (20), we obtain

$$
\left(1-\frac{c_{3}}{c_{1}}\right)\left(\frac{g^{n}(g-1)^{m} \prod_{j=1}^{d} g\left(q_{j} z+c_{j}\right)^{s_{j}}}{a(z)}\right)+\frac{1}{H}=1-\frac{c_{2}}{c_{1}}
$$

Using Lemma 3, we have

$$
\begin{aligned}
T\left(r, \frac{g^{n}(g-1)^{m} \prod_{j=1}^{d} g\left(q_{j} z+c_{j}\right)^{s_{j}}}{a(z)}\right) \leq & \bar{N}\left(r, \frac{g^{n}(g-1)^{m} \prod_{j=1}^{d} g\left(q_{j} z+c_{j}\right)^{s_{j}}}{a(z)}\right) \\
& +\bar{N}\left(r, \frac{a(z)}{g^{n}(g-1)^{m} \prod_{j=1}^{d} g\left(q_{j} z+c_{j}\right)^{s_{j}}}\right)+\bar{N}(r, H)+S(r, g)
\end{aligned}
$$

Next, we note that

$$
\begin{aligned}
T\left(r, g^{n}(g-1)^{m} \prod_{j=1}^{d} g\left(q_{j} z+c_{j}\right)^{s_{j}}\right) & \leq T\left(r, \frac{g^{n}(g-1)^{m} \prod_{j=1}^{d} g\left(q_{j} z+c_{j}\right)^{s_{j}}}{a(z)}\right)+T(r, a(z)) \\
& \leq T\left(r, \frac{g^{n}(g-1)^{m} \prod_{j=1}^{d} g\left(q_{j} z+c_{j}\right)^{s_{j}}}{a(z)}\right)+\log r
\end{aligned}
$$

By using (25), we get

$$
\begin{aligned}
T\left(r, g^{n}(g-1)^{m} \prod_{j=1}^{d} g\left(q_{j} z+c_{j}\right)^{s_{j}}\right) & \leq \bar{N}\left(r, \frac{g^{n}(g-1)^{m} \prod_{j=1}^{d} g\left(q_{j} z+c_{j}\right)^{s_{j}}}{a(z)}\right) \\
& +\bar{N}\left(r, \frac{a(z)}{g^{n}(g-1)^{m} \prod_{j=1}^{d} g\left(q_{j} z+c_{j}\right)^{s_{j}}}\right)+\bar{N}(r, H)+S(r, g) \\
& \leq(2 d+4) T(r, g)+S(r, g) .
\end{aligned}
$$

By Lemma 1 and (27), we have

$$
\begin{aligned}
(n+m) T(r, g) & =T\left(r, g^{n}(g-1)^{m}\right)+S(r, g) \\
& \leq T\left(r, g^{n}(g-1)^{m} \prod_{j=1}^{d} g\left(q_{j} z+c_{j}\right)^{s_{j}}\right)+T\left(r, \frac{1}{\prod_{j=1}^{d} g\left(q_{j} z+c_{j}\right)^{s_{j}}}\right)+S(r, g) \\
& \leq(2 d+4) T(r, g)+\lambda T(r, g)+S(r, g) \\
(n+m) T(r, g) & \leq(\lambda+2 d+4) T(r, g)+S(r, g) .
\end{aligned}
$$

Which is contradiction to $n>3 \lambda+m+2 d+7$. Hence our assumption neither $F_{2}$ nor $F_{3}$ is a constant is wrong. Hence one of the $F_{2}$ or $F_{3}$ is a constant. 
Case 4. Suppose that $F_{2}=c$, where $c(\neq 0)$ is a constant. If $c \neq 1$, then we have from (20)

$$
\frac{f^{n}(f-1)^{m} \prod_{j=1}^{d} f\left(q_{j} z+c_{j}\right)^{s_{j}}}{a(z)}-\frac{c g^{n}(g-1)^{m} \prod_{j=1}^{d} g\left(q_{j} z+c_{j}\right)^{s_{j}}}{a(z)}=1-c .
$$

Applying Lemma 3 to (28), we have

$$
\begin{aligned}
& T\left(r, \frac{f^{n}(f-1)^{m} \prod_{j=1}^{d} f\left(q_{j} z+c_{j}\right)^{s_{j}}}{a(z)}\right) \leq \bar{N}\left(r, \frac{f^{n}(f-1)^{m} \prod_{j=1}^{d} f\left(q_{j} z+c_{j}\right)^{s_{j}}}{a(z)}\right) \\
& +\bar{N}\left(r, \frac{a(z)}{f^{n}(f-1)^{m} \prod_{j=1}^{d} f\left(q_{j} z+c_{j}\right)^{s_{j}}}\right)+\bar{N}\left(r, \frac{-a(z)}{g^{n}(g-1)^{m} \prod_{j=1}^{d} g\left(q_{j} z+c_{j}\right)^{s_{j}}}\right)+S(r, f) \\
& \leq(d+1) \bar{N}(r, f)+(d+2) \bar{N}\left(r, \frac{1}{f}\right)+(d+2) \bar{N}\left(r, \frac{1}{g}\right)+S(r, f) .
\end{aligned}
$$

Next, we note that

$$
T\left(r, f^{n}(f-1)^{m} \prod_{j=1}^{d} f\left(q_{j} z+c_{j}\right)^{s_{j}}\right) \leq T\left(r, \frac{f^{n}(f-1)^{m} \prod_{j=1}^{d} f\left(q_{j} z+c_{j}\right)^{s_{j}}}{a(z)}\right)+\log r .
$$

Then from (29), we have

$$
T\left(r, f^{n}(f-1)^{m} \prod_{j=1}^{d} f\left(q_{j} z+c_{j}\right)^{s_{j}}\right) \leq(d+1) \bar{N}(r, f)+(d+2) \bar{N}\left(r, \frac{1}{f}\right)+(d+2) \bar{N}\left(r, \frac{1}{g}\right)+S(r, f) .
$$

By using Lemma 1 and (30), we have

$$
\begin{aligned}
(n+m) T(r, f)=T\left(r, f^{n}(f-1)^{m}\right)+S(r, f) & \leq T\left(r, f^{n}(f-1)^{m} \prod_{j=1}^{d} f\left(q_{j} z+c_{j}\right)^{s_{j}}\right)+T\left(r, \frac{1}{\prod_{j=1}^{d} f\left(q_{j} z+c_{j}\right)^{s_{j}}}\right)+S(r, f) \\
& \leq(d+1) \bar{N}(r, f)+(d+2) \bar{N}\left(r, \frac{1}{f}\right)+(d+2) \bar{N}\left(r, \frac{1}{g}\right)+\lambda T(r, f)+S(r, f) \\
& \leq(2 d+\lambda+3) T(r, f)+(d+2) T(r, g)+S(r, f) \\
(n+m-2 d-\lambda-3) T(r, f) & \leq(d+2) T(r, g)+S(r, f) .
\end{aligned}
$$

From Lemma 7, we have

$$
(n+m-2 d-\lambda-3) T(r, f) \leq\left(\frac{n+m+\lambda}{n+m-2 d-\lambda-3}\right)(d+2) T(r, f)+S(r, f) .
$$

Which is contradiction to $n \geq 3 \lambda+m+2 d+7$. Hence $c=1$. Then from (28), we have

$$
\Rightarrow f^{n}(f-1)^{m} \prod_{j=1}^{d} f\left(q_{j} z+c_{j}\right)^{s_{j}}=g^{n}(g-1)^{m} \prod_{j=1}^{d} g\left(q_{j} z+c_{j}\right)^{s_{j}} .
$$

Now we consider two subcases.

Subcase 4.1. If $m=1$, then from Lemma 8, we have $f=t g$ for a constant $t$ such that $t^{d}=1$ where $d=G C D(n+1+\lambda, n+\lambda)$. 
Subcase 4.2 If $m \geq 2$, let $h=\frac{f}{g}$ then $f=g h$, from (31), we have

$$
\begin{aligned}
& g^{n} h^{n}(g h-1)^{m} \prod_{j=1}^{d} g\left(q_{j} z+c_{j}\right)^{s_{j}} \prod_{j=1}^{d} h\left(q_{j} z+c_{j}\right)^{s_{j}}=g^{n}(g-1)^{m} \prod_{j=1}^{d} g\left(q_{j} z+c_{j}\right)^{s_{j}} \\
& \Rightarrow g^{n} h^{n}\left(g^{m} h^{m}-m g^{m-1} h^{m-1}+\frac{m(m-1)}{2} g^{m-2} h^{m-2}-\ldots+(-1)^{m}\right) \prod_{j=1}^{d} g\left(q_{j} z+c_{j}\right)^{s_{j}} \\
& \prod_{j=1}^{d} h\left(q_{j} z+c_{j}\right)^{s_{j}}-g^{n}\left(g^{m}-m g^{m-1}+\frac{m(m-1)}{2} g^{m-2}-\ldots+(-1)^{m}\right) \prod_{j=1}^{d} g\left(q_{j} z+c_{j}\right)^{s_{j}} \\
& \Rightarrow\left[g^{n+m}\left(h^{n+m} \prod_{j=1}^{d} h\left(q_{j} z+c_{j}\right)^{s_{j}}-1\right)-m g^{n+m-1}\left(h^{n+m-1} \prod_{j=1}^{d} h\left(q_{j} z+c_{j}\right)^{s_{j}}-1\right)+\right. \\
& \left.\ldots+(-1)^{m} g^{n}\left(h^{n} \prod_{j=1}^{d} h\left(q_{j} z+c_{j}\right)^{s_{j}}-1\right)\right] \prod_{j=1}^{d} g\left(q_{j} z+c_{j}\right)^{s_{j}}=0 .
\end{aligned}
$$

If $h$ is a constant, then $h^{d}=1$ where

$$
d=G C D(n+m+\lambda, n+m+\lambda-1, \ldots, n+m+\lambda-i, \ldots, n+\lambda)
$$

for $i=0,1,2, \ldots, m$. Hence $f=\operatorname{tg}$ for a constant $t$ such that $t^{d}=1$ where

$$
d=(n+m+\lambda, n+m+\lambda-1, \ldots, n+m+\lambda-i, \ldots, n+\lambda) .
$$

If $h$ is not a constant, then by (32) that $f$ and $g$ satisfy the algebraic equation $R(f, g) \equiv 0$, where

$$
R\left(\omega_{1}, \omega_{2}\right)=\omega_{1}^{n}\left(\omega_{1}-1\right)^{m} \prod_{j=1}^{d} \omega_{1}\left(q_{j} z+c_{j}\right)^{s_{j}}-\omega_{2}^{n}\left(\omega_{2}-1\right)^{m} \prod_{j=1}^{d} \omega_{2}\left(q_{j} z+c_{j}\right)^{s_{j}} .
$$

Case 5. Suppose that $F_{3}=k$, where $k(\neq 0)$ is a constant. If $k \neq 1$, then we have from (20)

$$
\frac{f^{n}(f-1)^{m} \prod_{j=1}^{d} f\left(q_{j} z+c_{j}\right)^{s_{j}}}{a(z)}-\frac{k a(z)}{g^{n}(g-1)^{m} \prod_{j=1}^{d} g\left(q_{j} z+c_{j}\right)^{s_{j}}}=1-k .
$$

Applying lemma 3, to the (33), we have

$$
\begin{aligned}
& T\left(r, \frac{f^{n}(f-1)^{m} \prod_{j=1}^{d} f\left(q_{j} z+c_{j}\right)^{s_{j}}}{a(z)}\right) \leq \bar{N}\left(r, \frac{f^{n}(f-1)^{m} \prod_{j=1}^{d} f\left(q_{j} z+c_{j}\right)^{s_{j}}}{a(z)}\right) \\
& +\bar{N}\left(r, \frac{a(z)}{f^{n}(f-1)^{m} \prod_{j=1}^{d} f\left(q_{j} z+c_{j}\right)^{s_{j}}}\right)+\bar{N}\left(r, \frac{g^{n}(g-1)^{m} \prod_{j=1}^{d} g\left(q_{j} z+c_{j}\right)^{s_{j}}}{a(z)}\right)+S(r, f) \\
& \leq(d+1) \bar{N}(r, f)+(d+2) \bar{N}\left(r, \frac{1}{f}\right)+(d+1) \bar{N}(r, g)+S(r, f) .
\end{aligned}
$$

Next, we note that

$$
T\left(r, f^{n}(f-1)^{m} \prod_{j=1}^{d} f\left(q_{j} z+c_{j}\right)^{s_{j}}\right) \leq T\left(r, \frac{f^{n}(f-1)^{m} \prod_{j=1}^{d} f\left(q_{j} z+c_{j}\right)^{s_{j}}}{a(z)}\right)+\log r
$$


From (34), we have

$$
T\left(r, f^{n}(f-1)^{m} \prod_{j=1}^{d} f\left(q_{j} z+c_{j}\right)^{s_{j}}\right) \leq(d+1) \bar{N}(r, f)+(d+2) \bar{N}\left(r, \frac{1}{f}\right)+(d+1) \bar{N}(r, g)+S(r, f) .
$$

By using Lemma 1 and (35), we have

$$
\begin{aligned}
(n+m) T(r, f) & =T\left(r, f^{n}(f-1)^{m}\right)+S(r, f) \\
& \leq T\left(r, f^{n}(f-1)^{m} \prod_{j=1}^{d} f\left(q_{j} z+c_{j}\right)^{s_{j}}\right)+T\left(r, \frac{1}{\prod_{j=1}^{d} f\left(q_{j} z+c_{j}\right)^{s_{j}}}\right)+S(r, f) \\
& \leq(d+1) \bar{N}(r, f)+(d+2) \bar{N}\left(r, \frac{1}{f}\right)+(d+1) \bar{N}(r, g)+\lambda T(r, f)+S(r, f) \\
& \leq(2 d+\lambda+3) T(r, f)+(d+1) T(r, g)+S(r, f) \\
(n+m-2 d-\lambda-3) T(r, f) & \leq(d+1) T(r, g)+S(r, f) .
\end{aligned}
$$

From Lemma 7, we have

$$
(n+m-2 d-\lambda-3) T(r, f) \leq\left(\frac{n+m+\lambda}{n+m-2 d-\lambda-3}\right)(d+1) T(r, f)+S(r, f)
$$

Which is contradiction to $n \geq 3 \lambda+m+2 d+7$. Therefore $k=1$ and by (17) and (20) we have $F_{1}+F_{2}=0$

$$
\begin{aligned}
& \Rightarrow \frac{f^{n}(f-1)^{m} \prod_{j=1}^{d} f\left(q_{j} z+c_{j}\right)^{s_{j}}}{a(z)}-\frac{a(z)}{g^{n}(g-1)^{m} \prod_{j=1}^{d} g\left(q_{j} z+c_{j}\right)^{s_{j}}}=0 \\
& \Rightarrow f^{n}(f-1)^{m} \prod_{j=1}^{d} f\left(q_{j} z+c_{j}\right)^{s_{j}} g^{n}(g-1) \prod_{j=1}^{d} g\left(q_{j} z+c_{j}\right)^{s_{j}}=a^{2}(z) \\
& \Rightarrow f^{n}(f-1)^{m} g^{n}(g-1)=\frac{a^{2}(z)}{\prod_{j=1}^{d} f\left(q_{j} z+c_{j}\right)^{s_{j}} \prod_{j=1}^{d} g\left(q_{j} z+c_{j}\right)^{s_{j}}} .
\end{aligned}
$$

From second fundamental theorem, we have

$$
\begin{aligned}
T(r, F) & \leq \bar{N}(r, F)+\bar{N}\left(r, \frac{1}{F}\right)+\bar{N}\left(r, \frac{1}{F-a(z)}\right)+S(r, F) \\
& \leq \bar{N}\left(r, f^{n}(f-1)^{m} \prod_{j=1}^{d} f\left(q_{j} z+c_{j}\right)^{s_{j}}\right)+\bar{N}\left(r, \frac{1}{f^{n}(f-1)^{m} \prod_{j=1}^{d} f\left(q_{j} z+c_{j}\right)^{s_{j}}}\right) \\
& +\bar{N}\left(r, \frac{1}{g^{n}(g-1)^{m} \prod_{j=1}^{d} g\left(q_{j} z+c_{j}\right)^{s_{j}}-z}\right)+S(r, f) \\
& \leq \bar{N}(r, f)+\bar{N}\left(r, \prod_{j=1}^{d} f\left(q_{j} z+c_{j}\right)^{s_{j}}\right)+\bar{N}\left(r, \frac{1}{f^{n}}\right) \\
& +\bar{N}\left(r, \frac{1}{(f-1)^{m}}\right)+\bar{N}\left(r, \frac{1}{\prod_{j=1}^{d} f\left(q_{j} z+c_{j}\right)^{s_{j}}}\right)+T(r, G)+S(r, f)
\end{aligned}
$$


and

$$
\begin{aligned}
(n+m+\lambda) T(r, f) & \leq(3+2 d) T(r, f)+(n+m+\lambda) T(r, g)+S(r, f) \\
(n+m+\lambda-2 d-3) T(r, f) & \leq(n+m+\lambda) T(r, g)+S(r, f) \\
T(r, f) & \leq\left(\frac{n+m+\lambda}{n+m+\lambda-2 d-3}\right) T(r, g)+S(r, f) .
\end{aligned}
$$

Therefore

$$
T(r, f)=O(T(r, g))
$$

Similarly, we have $T(r, g)=O(T(r, f))$. From (36), we have

$$
\begin{aligned}
(n+m)[N(r, f)+N(r, g)] & \leq N\left(r, a^{2}(z)\right)+N\left(r, \frac{1}{\prod_{j=1}^{d} f\left(q_{j} z+c_{j}\right)^{s_{j}}}\right)+N\left(r, \frac{1}{\prod_{j=1}^{d} g\left(q_{j} z+c_{j}\right)^{s_{j}}}\right) \\
& \leq \lambda N\left(\left(r, \frac{1}{f}\right)+\lambda N\left(r, \frac{1}{g}\right)+S(r, f)+S(r, g) .\right.
\end{aligned}
$$

Since

$$
\frac{1}{f^{n}(f-1)^{m} g^{n}(g-1)^{m}}=\frac{\prod_{j=1}^{d} f\left(q_{j} z+c_{j}\right)^{s_{j}} \prod_{j=1}^{d} g\left(q_{j} z+c_{j}\right)^{s_{j}}}{a^{2}(z)}
$$

We obtain

$$
\begin{aligned}
& n\left[N\left(r, \frac{1}{f}\right)+N\left(r, \frac{1}{g}\right)\right]+N\left(r, \frac{1}{(f-1)^{m}}\right)+N\left(r, \frac{1}{(g-1)^{m}}\right) \\
& \leq N\left(r, \prod_{j=1}^{d} f\left(q_{j} z+c_{j}\right)^{s_{j}}\right)+N\left(r, \prod_{j=1}^{d} g\left(q_{j} z+c_{j}\right)^{s_{j}}\right)+N\left(r, \frac{1}{a^{2}(z)}\right) \\
& \leq \lambda(N(r, f)+N(r, g))+S(r, f)+S(r, g) .
\end{aligned}
$$

From (37) and (38), we have

$$
\begin{aligned}
& (n+m)[N(r, f)+N(r, g)]+n\left[N\left(r, \frac{1}{f}\right)+N\left(r, \frac{1}{g}\right)\right]+N\left(r, \frac{1}{(f-1)^{m}}\right) \\
& +N\left(r, \frac{1}{(g-1)^{m}}\right)-\lambda N\left(r, \frac{1}{f}\right)-\lambda N\left(r, \frac{1}{g}\right)-\lambda N(r, f)-\lambda N(r, g) \leq S(r, f)+S(r, g) \\
& \Rightarrow N(r, f)+N(r, g)+N\left(r, \frac{1}{f}\right)+N\left(r, \frac{1}{g}\right)+N\left(r, \frac{1}{(f-1)}\right)+N\left(r, \frac{1}{(g-1)}\right) \leq S(r, f)+S(r, g)
\end{aligned}
$$

By second fundamental theorem and (39), we have

$$
T(r, f) \leq \bar{N}(r, f)+\bar{N}\left(r, \frac{1}{f}\right)+\bar{N}\left(r, \frac{1}{(f-1)}\right)+O(\log r) \leq o(T(r, f))
$$

which is contradiction. Hence $F_{3}$ is not a constant.

\section{Competing interests}

The authors declare that they have no competing interests. 


\section{Authors' contributions}

All authors have contributed to all parts of the article. All authors read and approved the final manuscript.

\section{References}

[1] R. S. Dyavanal and R. V. Desai, Uniqueness of difference polynomials of entire functions, Appl. J. Math., 869(2014): 3419-3424.

[2] R. S. Dyavanal and R. V. Desai, Uniqueness of q-shift difference and differential polynomials of entire functions, Far East J. Appl. Math., 91(3)(2015): 189-202.

[3] R. S. Dyavanal and A. M. Hattikal, Uniqueness of difference-differential polynomials of entire functions sharing one value, Tamkang J. Math., 47(2)(2016): 193-206.

[4] R. S. Dyavanal and A. M. Hattikal, Weighted sharing of uniqueness of difference polynomials of meromorphic functions, Far East J. Math. Sci., 98(3)(2015): 293-313.

[5] R. S. Dyavanal and A. M. Hattikal, Unicity theorems on difference polynomials of meromorphic functions sharing one value, Int. J. Pure Appl. Math. Sci., 9(2)(2016): 89-97.

[6] R. S. Dyavanal, A.M. Hattikal, On the uniqueness of product of difference polynomials of meromorphic functions, Konuralp J. Math. 4(2) (2016): 42-55.

[7] W.K.Hayman, Meromorphic functions, Claredon Press, Oxford, (1964).

[8] X.M.Li, H.X.Yi, W.L.Li, Value distribution of certain difference polynomials of meromorphic functions, Rocky Mountain J. Math., (2014),44(2): 599-632.

[9] Y.Liu, Y.H.Cao, X.G.Qi, H.X.Yi, Value sharing results for q-shifts difference polynomials, Discrete Dyn. Nat. Soc. 2013, Article ID 152069, 6 pages.

[10] F.H.Liu, H.X.Yi, Uniqueness of meromorphic function concerning the difference polynomials, Kyungpook Math. J. 55(2015): 411-427.

[11] H.Y.Xu, B.X.Liu, K.Z.Tang, Some properties of meromorphic solutions of systems of complex q-shift difference equations, Abstr. Appl. Anal. 2013, Article ID 680956, 6 pages.

[12] J.F.Xu, H.X.Yi, F.Lu Fixed points and uniqueness of meromorphic functions, Comp. Math. Appl. 59(2010): 9-17.

[13] L.Yang, Value Distribution Theory, Springer-Verlag Berlin, (1993).

[14] C.C.Yang, H.X.Yi, Uniqueness Theory of Meromorphic Functions, Kluwer Academic Publishers, Dordrecht,2003; Chinese original: Science Press, Beijing, (1995).

[15] H.X.Yi, Uniqueness of meromorphic functions and a question of C.C.Yang, Complex Var. 14(1990): 69-176.

[16] K.Zhang, H.X.Yi, The value distribution and uniqueness of one certain type of differential-difference polynomials, Acta Mathematica Sci. 34B(3)(2014): 719-728. 